OPEN ACCESS

Edited by: Diamant Thaci, University of Lübeck, Germany

Reviewed by:

Marcus G. Tan, University of Ottawa, Canada Takashi Hashimoto, Osaka City University, Japan

*Correspondence: Koichiro Nakamura nakamu@saitama-med.ac.jp

Specialty section: This article was submitted to Dermatology

a section of the journal

Frontiers in Medicine

Received: 02 October 2020 Accepted: 30 December 2020 Published: 01 February 2021

Citation:

Nakamura K, Tsunemi Y, Kaneko F and Alpsoy E (2021) Mucocutaneous Manifestations of Behçet's Disease.

Front. Med. 7:613432.

doi: 10.3389/fmed.2020.613432

\section{Mucocutaneous Manifestations of Behçet's Disease}

\author{
Koichiro Nakamura ${ }^{1 *}$, Yuichiro Tsunemi ${ }^{1}$, Fumio Kaneko ${ }^{2}$ and Erkan Alpsoy ${ }^{3}$ \\ ${ }^{1}$ Department of Dermatology, Saitama Medical University, Saitama, Japan, ${ }^{2}$ Institute of Dermato-Immunology and Allergy, \\ Southern Tohoku General Hospital, Fukushima, Japan, ${ }^{3}$ Department of Dermatology and Venereology, School of Medicine, \\ Akdeniz University, Antalya, Turkey
}

Behçet's disease $(\mathrm{BD})$ is a chronic, relapsing, systemic inflammatory disease with clinical features showing mucocutaneous lesions involving the ocular, articular, and further miscellaneous organs. Mucocutaneous manifestations, one of the most characteristic signs of BD, have been most commonly observed upon onset or at any disease stage and are exceptionally important in its diagnosis. Given the lack of specific diagnostic laboratory tests for $\mathrm{BD}$, diagnosis has been based on clinical findings. All diagnostic criteria published have thus far relied heavily on mucocutaneous manifestations, particularly oral ulcers $(\mathrm{OU})$, genital ulcers $(\mathrm{GU})$, cutaneous lesions, and pathergy test positivity. Worldwide, OU, GU, cutaneous lesions, and ocular and articular manifestations have been the most common symptoms, with erythema nodosum (EN)-like lesions and papulopustular lesions being the most prevalent cutaneous manifestations. While majority of the patients worldwide have reported $\mathrm{OU}$ as the most frequent symptom upon disease onset, GU, and EN-like lesions have also been identified upon onset. Considering that mucocutaneous symptoms precede severe organ involvement in most patients, familiarity with such symptoms is imperative for early diagnosis and prevention of potentially serious organ involvement through appropriate management.

Keywords: Behçet's disease, erythema nodosum-like lesion, papulopustular lesion, oral ulcer, genital ulcer

\section{INTRODUCTION}

Behçet's disease $(\mathrm{BD})$ is a chronic, relapsing, systemic inflammatory disease of unknown etiology that is clinically characterized by oral ulcers (OU), genital ulcers (GU), cutaneous lesions, and ocular symptoms.

However, specific clinical types with vascular, gastrointestinal, and central nervous system involvement have also been reported (1-4). Given the lack of definitive diagnostic laboratory and histopathological tests for the disease, diagnosis has solely been based on clinical findings. The various diagnostic criteria used in diagnosing this disease have commonly included mucocutaneous lesions, particularly OU, GU, cutaneous lesions, and pathergy test positivity (5). The most extensively utilized set of diagnostic criteria has been established by the International Study Group for Behçet's Disease criteria, among three of the five findings (OU, GU, cutaneous lesions, ocular involvement, and pathergy test positivity) comprise mucocutaneous lesions including OU, GU, and cutaneous lesions (6). The Japanese Diagnostic Criteria, one of the most sensitive and specific criteria, have been used particularly in Far Eastern countries (2), among which 
three of the four major criteria (OU, GU, cutaneous lesions, and ocular involvement) include mucocutaneous symptoms.

Mucocutaneous lesions have been the most common symptoms upon onset or at any stage of BD. Accordingly, OU (92-100\%) and GU (57-93\%), cutaneous lesions (38$99 \%)$, and ocular (29-100\%) and articular manifestations (16-84\%) have been the most common symptoms observed globally, with erythema nodosum (EN)-like lesions (15-78\%) and papulopustular lesions (PPL) (28-96\%) being the most frequently documented cutaneous manifestations. While OU have been the most frequently observed symptoms upon disease onset in majority of the patients worldwide, GU and ENlike lesions have also been reported at onset (7). Previous studies have demonstrated that a significant proportion of patients, only present with mucocutaneous symptoms (8, 9). However, the absence of major organ involvement in the early years of $\mathrm{BD}$ does not suggest a mild prognosis, particularly for young male patients. Considering that in most instances, mucocutaneous lesions appear before serious organ involvement develops. Therefore, familiarity with the mucocutaneous spectrum is imperative for early diagnosis and treatment, which would prevent most of the hazardous consequences of BD.

The current review will discuss the latest evidence regarding mucocutaneous manifestations of BD.

\section{ORAL ULCERS}

OU, one of the major initial signs of the disease, have been found in $97-99 \%$ of patients according to data obtained by the International Study Group for Behçet's Disease from several countries (6). OU typically occurs at an average of 7 years before the diagnosis. Bang et al. prospectively followed 67 cases with recurrent aphthous stomatitis (RAS) and observed that 35 (52.2\%) patients developed BD symptoms after an average of 7.7 years. Similarly, they found the annual frequency of the lesions to be 9.8 in progressive patients (10).

Patients with OU initially present with a pseudo-membrane in the center surrounded by a reddish halo, shortly reaching the central ulcer. OU generally appear on the lips, buccal mucosa, gingiva, and tongue, with lesions occasionally developing on the palate. Albeit rarely, OU may occur on the tonsil and pharynx and cause considerable discomfort and resistance to conventional treatment modalities.

Minor OU are small and shallow ulcers, usually 3-6 mm $(<10 \mathrm{~mm})$ in diameter. Although minor OU, seen in about $80 \%$ of patients, heal rapidly with no scarring, OU regress and recur repeatedly over a prolonged period. Major OU are large and deep ulcers, having diameter more than $10-30 \mathrm{~mm}$ and have severe pain (Figure 1A). While major OU are morphologically similar to minor OU, they are larger $(>1 \mathrm{~cm})$, deeper, and more painful. Moreover, such ulcers last longer and often heal with scarring and tissue loss. Notably, major OU, which occur frequently in patients with $\mathrm{BD}$, have been one of the important parameters in distinguishing BD from RAS. In a comparative study of 1,643 and 3,527 patients with RAS and $\mathrm{BD}$, respectively. Oh et al. reported that major OU were significantly more common and affected more oral mucosa regions in patients with $\mathrm{BD}(11)$.

Although OU alone have limited diagnostic value, such value increases in the presence of GU, uveitis, etc. However, OU have been the most common reasons for referral to the outpatient clinic and are one of the most active symptoms of $\mathrm{BD}$, with recurrent periods of exacerbation and remission. Accordingly, a cross-sectional multicenter study, observed OU activity in $65.1 \%$ of the study group, and found that OU activity was associated with non-immunosuppressive use in both genders, as well as early disease in males and resistant cases in females (12). As such, suppression of OU activity is a crucial and common practice in the management of $\mathrm{BD}$.

The appearance of OU often causes quality of life (QOL) disturbances. Accordingly, a study examining the 1-year prevalence of $\mathrm{OU}$ and its association with oral health-related QOL in patients with $\mathrm{BD}$ and general population found that those with $\mathrm{BD}$ who had OU (odds ratio, 6.2; $n=529$ ) had more disturbed QOL compared to general population without OU (odds ratio, 1.0; $n=1,097$ ) (13).

\section{GENITAL ULCERS}

$\mathrm{GU}$, another characteristic feature of $\mathrm{BD}$, may serve as the initial sign of the disease. Although the appearance and course of GU are similar to those of OU, they are generally deeper and less often recurring (Figure 1B). Given that deep-seated ulcers may heal with scarring, scars of previous lesions should be investigated in patients suspected to have $\mathrm{BD}$, despite the absence of $\mathrm{GU}$ at that time. Mat et al. who investigated the frequency of scarring in GU (14), found that $89 \%$ of large ulcers $(\geq 1 \mathrm{~cm})$ and $49 \%$ of small ulcers $(<1 \mathrm{~cm})$ healed with scarring in male patients. Meanwhile, the rate of scar formation in female patients with large and small GU was 100 and $40.5 \%$, respectively. The aforementioned study observed that ulcers located in the labium minus and vestibule can heal without scarring. Therefore, scars from previous lesions should be investigated apart from active lesions in the diagnosis of BD.

Approximately $90 \%$ of $\mathrm{GU}$ in men develop on the scrotum, with lesions rarely locating on the glans and corpus penis. In women, the most common localization is the labium, although they develop in the vulva, vagina, and even cervix. Bloodypurulent discharge, pain, and dyspareunia may be observed due to its location in the vagina. Moreover, vulval ulcers can occasionally lead to tissue loss in the labia. Although rare, deeply located ulcers may fistulize into the bladder, urethra, and rectum. Furthermore, reports have been shown perianal region and inguinal fold localization in both sexes (15).

In summary, GU are one of the three symptom complexes originally characterizing the disease. Compared to OU, GU are more specific for the diagnosis of BD. The International Study Group for Behçet's Disease compared the data of 914 patients with Behçet's disease from 12 centers in 7 countries with those of controls in the same centers. In a new diagnostic criterion, the sensitivity and specificity of each BD symptom were determined and relative value and expected value were calculated. Of all BD 

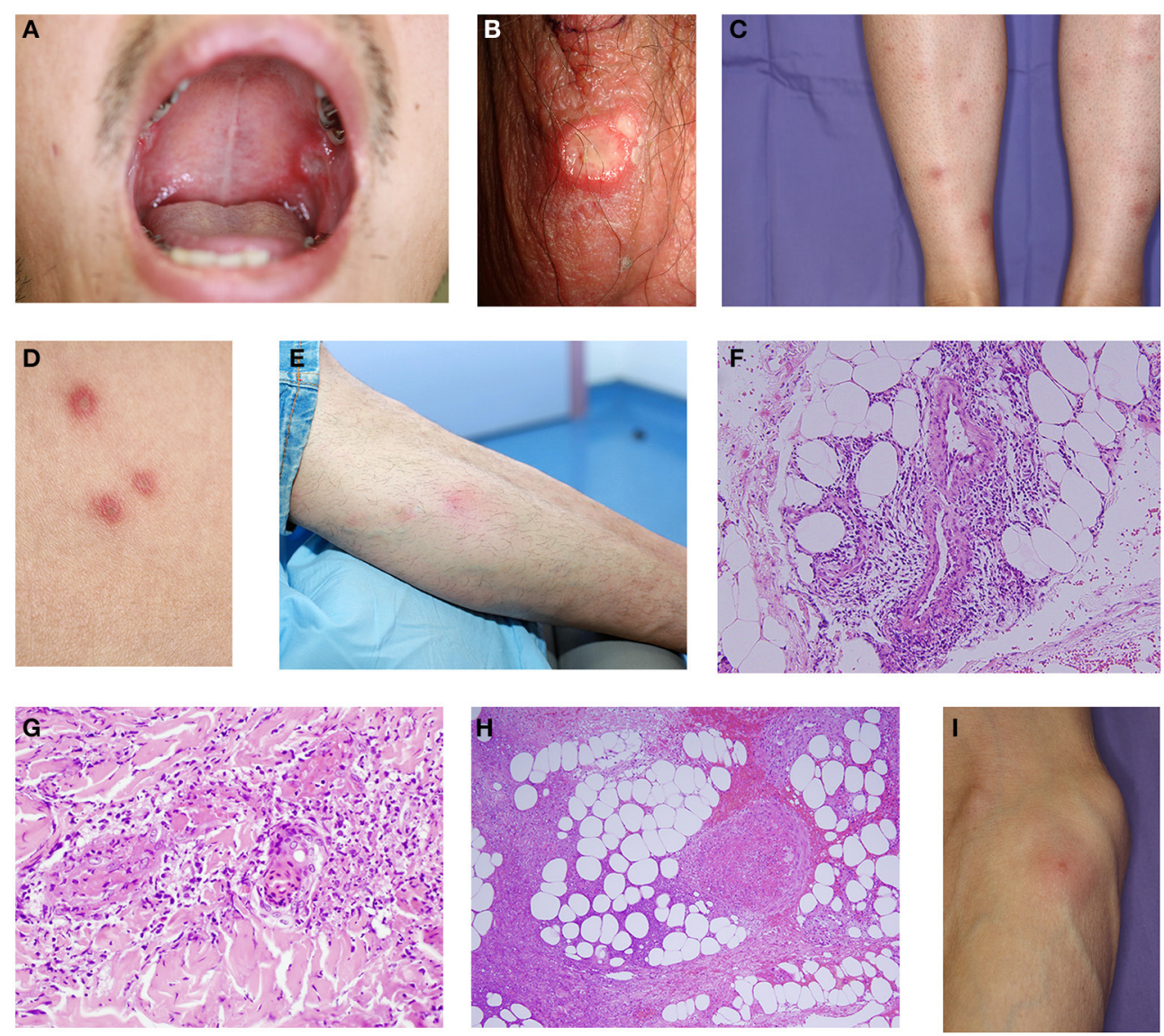

FIGURE 1 | (A) Oral ulcer on the buccal mucosa with rolled borders and grayish necrotic base. (B) Genital ulcer with yellowish necrotic tissue on the scrotum. The genital ulcer is deep and surrounded by erythema. The scrotum is the site most commonly involved in the male. (C) Erythema nodosum-like lesions on the lower leg. A painful, reddish erytemanous nodule is detected in the pretibial lesion. (D) Papulopustular folliculitis are sterile pustules on the erythematous base of the trunk. (E) Superficial thrombophlebitis is erythematous tender subcutaneous nodules arranged in a linear fashion on the leg. (F) Histological feature of erythema nodosum-like lesion includes a dense neutrophil infiltrate around the vessels in the subcutaneous tissue. (G) Histological feature of papulopustular lesion includes a leukocytoclastic vasculitis with neutrophil infiltrate in the dermis. (H) Histology of thrombophlebitis shows vascular thrombus and lymphocytic infiltrate in a subcutaneous vein. (I) Skin pathergy reaction is a pustule formation at the needle site after $24 \mathrm{~h}$ on the forearm. (A,F,H,I) are derived from Nakamura et al. $(3,4)$.

symptoms, GU had the highest relative value and expected value (6). The presence of recurrent $\mathrm{OU}$ and GU are strongly evocative of $\mathrm{BD}$, especially in endemic regions.

\section{CUTANEOUS LESIONS}

EN-like lesions, PPL and superficial thrombophlebitis represent the most characteristic cutaneous lesions of BD. Moreover, cutaneous lesions are among the first symptoms of $\mathrm{BD}$ and often show a course with recurrent attacks (7). Therefore, cutaneous lesions need to be diagnosed in the early stages of $\mathrm{BD}$. One study showed that among patients with BD in Japan, 88.8\% (513 of 578 patients) exhibited cutaneous lesions (16).

\section{Erythema Nodosum (EN)-Like Lesions}

EN-like lesions are painful, tender, oval, erythematous nodules that often develop on the legs (Figure 1C) and are more common in females. Although most frequently observed in the lower extremities, they can also be located in other body parts, such as the gluteal region, upper extremities, face, and neck. The lesions regress within 2-3 weeks with local pigmentation and sometimes scaling, especially in dark-skinned individuals. Several patients with EN-like lesions have accompanying systemic symptoms, such as joint symptoms, malaise, and fever.

Although EN-like lesions are characteristic of $\mathrm{BD}$, these lesions are not specific to the disease. Thus, differentiating between EN-like lesions of $\mathrm{BD}$ and classic EN, which is caused by bacterial or viral infections, such as streptococcal pharyngitis, is necessary. Other differentiate conditions include EN accompanied by ulcerative colitis, Sweet's syndrome, and Crohn's disease. A skin biopsy is useful to confirm the inflammatory and vascular changes seen in EN-like lesions of BD.

\section{Papulopustular Lesions}

PPL, observed in $65-96 \%$ of the cases, have been the most common cutaneous manifestation of the disease. They are 
characterized by folliculitis or acne-like sterile papulopustules on an erythematous base (Figure 1D). Typical lesions appear as papules that develop pustules within $24-48 \mathrm{~h}$ and are often localized in the trunk, lower extremities, and face (17). Moreover, PPL have been frequently reported in patients with arthritis (18).

BD is usually diagnosed between the ages of 20-30 during which acne vulgaris is also relatively common. Distinguishing between PPL in BD from acne vulgaris is extremely important in patients diagnosed with $\mathrm{BD}$ in the presence of PPL. We believe that follicular PPL is not specific to the disease. PPL in $\mathrm{BD}$ include follicular and non-follicular lesions. Follicular lesion of $\mathrm{BD}$ sometimes includes a hair follicle, namely a pustule containing hair designed as a folliculitis. However, Boyvat et al. have observed neutrophilic vasculitis in cases with non-follicular PPL (19) and this observation supports the notion that nonfollicular PPL is more specific to the clinical characteristic feature of BD (Figures 1D,G). Therefore, considering only non-follicular PPL would be more appropriate. Again, localization in nonseborrheic areas, such as the lower extremities, can be more specific for $\mathrm{BD}$ and should be preferred as a biopsy site.

\section{Superficial Thrombophlebitis}

Superficial thrombophlebitis is a palpable induration along the course of a vein, mainly in the legs (Figure 1E) that occurs more frequently in male cases and most frequently affects the vena saphena magna. This condition may present clinically as superficial migratory thrombophlebitis. Clinically distinguishing between superficial thrombophlebitis and ENlike lesions may be difficult when a short vein segment is involved. Nonetheless, ultrasonography can help differentiate both conditions in addition to clinical examination, with dermal ultrasonography, revealing a hyperechoic pattern in EN-like lesions, but a hypoechoic pattern in superficial thrombophlebitis.

Although superficial thrombophlebitis can be detected during dermatological examination, it indicates vascular involvement and closely associated with deep vein thrombosis and dural sinus thrombosis (20). Close follow-up of cases with superficial thrombophlebitis should therefore be appropriate in terms of vascular involvement.

\section{Histopathology of Cutaneous Lesions}

Histologically, early-stage EN-like lesions are characterized by septal panniculitis accompanied by a marked neutrophilic infiltrate, particularly around blood vessels in the upper and lower dermis, and extravascular leakage of red blood cells (Figure 1F). Lymphocytic infiltrates predominate in addition to neutrophils in the late stage. Occasionally, a vascular thrombus can occur as inflammatory changes. The existence of neutrophilic perivascular infiltration, sometimes vasculitis with neutrophil infiltration, is a pathological finding of $\mathrm{EN}$ of $\mathrm{BD}$, which is an important feature differentiating from classical EN.

In histopathological examination of papulopustular lesion, pustules exhibit neutrophilic infiltrates around the hair follicles and just below the epidermis. Neutrophils also infiltrate around the blood vessels in the dermis. One study showed that leukocytoclastic vasculitis in $10(43.5 \%)$ of 23 patients with pustular lesions (19). Meanwhile, Alpsoy et al. reported that 12
(70.5\%) of 17 patients with BD exhibited 11 leukocytoclastic vasculitis and 1 lymphocytic vasculitis (21). Moreover, Ilknur et al. reported that $27.5 \%$ of patients with $\mathrm{BD}$ experienced vasculitis, whereas none of the controls showed the same (22). However, Ergun et al. reported that PPL do not affect the development of vasculitis (23). Finally, Jorizzo et al. reported the importance of histologic changes in non-follicular PPL for the diagnosis of BD (24). Given that vascular histopathological changes may be specific for PPL, we strongly suggest considering the presence of leukocytoclastic vasculitis or neutrophilic vascular reaction in the histology of non-follicular PPL as a diagnostic criterion (21) (Figure 1G).

Histologically, stenosis and thrombus formation have been observed in the lumen of a superficial subcutaneous vein along with neutrophil and lymphocytic infiltrates of superficial thrombophlebitis (Figure 1H). The Chapel Hill Consensus Conference indicated that BD is involved in variable-sized vessel vasculitis (25). Another study showed that among 2,319 patients, $14.3 \%$ had vascular involvement, while $53.3 \%$ had superficial thrombophlebitis, indicating that superficial thrombophlebitis was the most common feature of vascular change (26).

\section{Other Cutaneous Lesions}

Other common and well-known skin manifestations of $\mathrm{BD}$ include extragenital ulcers and Sweet's syndrome-like lesions.

Extragenital ulcers, which are clinically similar to aphthous lesions and are one of the most specific symptoms of $\mathrm{BD}$, are deep-seated, punched-out ulcers with erythematous and edematous edges and a yellow necrotic base. Extragenital ulcers follow a recurrent course, can usually result in scarring, and can be localized to the legs, armpits, breast, neck, toes, inguinal region, and neck. Meanwhile, Sweet's syndromelike lesion is a painful reddish plaque usually observed in the face, neck, and extremities and rarely associated with BD. Other lesions (e.g., pyoderma gangrenosumlike lesions, erythema multiforme-like lesions, pernio-like cutaneous lesions, palpable purpura, Henoch-Schönlein purpura, bullous necrotizing vasculitis, subungual infarctions, hemorrhagic bullae, furuncles, abscesses, and acral purpuric papulonodular lesions) have been limited to case reports. Histologically, neutrophilic infiltrates have been observed in the skin of all such lesions and are therefore referred to as neutrophilic dermatoses.

\section{SKIN PATHERGY TEST}

A pathergy test determined the hypersensitivity reaction after a needle puncture (Figure 1I), with positivity being defined as the development of an erythematous papule or pustule around the injection site $24-48 \mathrm{~h}$ after intradermal puncture of the skin. While $\sim 50 \%$ of the patients in the Middle and Near East have pathergy test positivity, $\sim<30 \%$ in Japan have the same. Numerous factors affect the positivity rate of skin pathergy tests, including the thickness of the needle used, sharpness or blunt tip of the needle, number of punctures, method of application, disinfection of the test site with an antiseptic, etc, which might explain the differences in pathergy test positivity 
among these countries. A positive result to a pathergy test is a major criterion in the International Criteria for Behçet's Disease and International Study Group for Behçet's Disease and is an important manifestation for early diagnosis.

\section{CONCLUSION}

We hereline discussed clinical features of mucocutaneous lesions observed in BD. Accordingly, we highlight that mucocutaneous lesions precede the diagnosis of $\mathrm{BD}$. Moreover, given that patients can show variable symptoms during their course, excluding other disease is always necessary to ensure correct diagnosis. Characteristic feature of $\mathrm{BD}$, such as $\mathrm{OU}, \mathrm{GU}, \mathrm{EN}$-like lesions, PPL, superficial thrombophlebitis, and skin pathergy test are manifestations that can be used for early diagnosis. Considering

\section{REFERENCES}

1. Yazici H, Seyahi E, Hatemi G, Yazici Y. Behçet's disease: a contemporary view. Nat Rev Rheumatol. (2018) 14:107-19. doi: 10.1038/nrrheum.2017.208

2. Sakane T, Takeno M, Suzuki N, Inaba G. Behçet's disease. N Eng J Med. (1999) 341:1284-91. doi: 10.1056/NEJM199910213411707

3. Nakamura K, Iwata Y, Asai J, Kawakami T, Tsunemi Y, Takeuchi M, et al. Guidelines for the treatment of skin and mucosal lesions in Behçet's disease: a secondary publication. J Dermatol. (2020) 47:22335. doi: 10.1111/1346-8138.15207

4. Nakamura K, Iwata Y, Asai J, Kawakami T, Tsunemi Y, Takeuchi M, et al. Clinical Features and Algorithm of Treatment. 2020 JSBD Clinical Practice Guidelines for Behçet's Disease. Mizuki N, Takeuchi M, editors. Tokyo: Shindan To Chiro Sha, Inc. (2020). p. 20-3, p. 54-6.

5. Alpsoy E. Behçet's disease: a comprehensive review with a focus on epidemiology, etiology and clinical features, and management of mucocutaneous lesions. J Dermatol. (2016) 43:620-32. doi: 10.1111/1346-8138.13381

6. International Study Group for Behçet's Disease. Criteria for diagnosis of Behçet's disease. Lancet. (1990) 335:107880. doi: 10.1016/0140-6736(90)92643-V

7. Alpsoy E, Donmez L, Onder M, Gunasti S, Usta A, Karincaoglu $\mathrm{Y}$, et al. Clinical features and natural course of Behcet's disease in 661 cases: a multicentre study. $\mathrm{Br} J$ Dermatol. (2007) 157:901-06. doi: 10.1111/j.1365-2133.2007.08116.x

8. Ugurlu N, Bozkurt S, Bacanli A, Akman-Karakas A, Uzun S, Alpsoy E. The natural course and factors affecting severity of Behçet's disease: a single-center cohort of 368 patients. Rheumatol Int. (2015) 35:210307. doi: 10.1007/s00296-015-3310-5

9. Hamuryudan V, Hatemi G, Tascilar K, Sut N, Ozyazgan Y, Seyahi E, et al. Prognosis of Behçet's syndrome among men with mucocutaneous involvement at disease onset: long-term outcome of patients enrolled in a controlled trial. Rheumatology. (2010) 49:173-77. doi: 10.1093/rheumatology/kep350

10. Bang D, Yoon KH, Chong HO, Choi EH, Lee E-S, Lee S. Epidemiological and clinical features of Behçet's disease in Korea. Yonsei Med J. (1997) 38:428-36. doi: 10.3349/ymj.1997.38.6.428

11. Oh SH, Han EC, Lee JH, Bang D. Comparison of the clinical features of recurrent aphthous stomatitis and Behcet's disease. Clin Exp Dermatol. (2009) 34:208-12. doi: 10.1111/j.1365-2230.2009.03 384.x

12. Mumcu G, Karacayli Ü, Yay M, Aksoy A, Tas MN, Amagan B, et al. Oral ulcer activity assessment with the composite index according to different treatment modalities in Behçet's syndrome: a multicentre study. Clin Exp Rheumatol. (2019) 121(37 Suppl):98-104.

13. Naito M, Suzukamo Y, Wakai K, Azechi M, Kaneko F, Nakayama T, et al. One-year period prevalence of oral aphthous ulcers and oral healthy-related that patients often show poor QOL, early diagnosis and treatment initiation with careful observation of the course is favorable.

\section{AUTHOR CONTRIBUTIONS}

$\mathrm{KN}, \mathrm{YT}, \mathrm{FK}$, and EA: writing and revision of the manuscript. $\mathrm{KN}, \mathrm{YT}$, and EA: acquisition of clinical data. EA: conception and design and study supervision. All authors contributed to the article and approved the submitted version.

\section{ACKNOWLEDGMENTS}

This study was in part supported by Grant-in-Aid for Scientific Research from Ministry of Health, Labor, and Welfare (20FC1012) and by AMED (20ek0109473s0801) in Japan.

quality of life in patients with Behçet's disease. Genet Res Int. (2014) 2014:930348. doi: 10.1155/2014/930348

14. Mat C, Goksugur N, Ergin B, Yurdakul S, Yazici H. The frequency of scarring after genital ulcers in Behcet's syndrome: a prospective study. Int J Dermatol. (2006) 45:554-6. doi: 10.1111/j.1365-4632.2006.02859.x

15. Alpsoy E, Zouboulis CC, Ehrlich CE. Mucocutaneous lesions of Behçet's disease. Yonsei Med J. (2007) 48:573-85. doi: 10.3349/ymj.2007.48. 4.573

16. Kirino Y, Ideguchi H, Takeno M, Suda A, Higashitani K, Kunishita Y, et al. Continuous evolution of clinical phenotype in 578 Japanese patients with Behçet's disease: a retrospective observational study. Arthritis Res Ther. (2016) 18:217. doi10.1186/s13075-016-1115-X

17. Alpsoy E, Aktekin M, Er H, Durusoy C, Yilmaz E. A randomized, controlled and blinded study of papulopustular lesions in Turkish Behcet's patients. Int J Dermatol. (1998) 37:839-3. doi: 10.1046/j.1365-4362.1998. 00401.x

18. Diri E, Mat C, Hamuryudan V, Yurdakul S, Hizli N, Yazici. Papulopustular skin lesions are seen more frequently in patients with Behçet's syndrome who have arthritis: a controlled and masked study. Ann Rheum Dis. (2001) 60:1074-76. doi: 10.1136/ard.60.11.1074

19. Boyvat A, Heper AO, Kocyigit P, Erekul S, Gurgey E. Can specific vesselbased papulopustular lesions of Behçet's disease be differentiated from nonspecific follicular-based lesions clinically? Int J Dermatol. (2006) 45:8148. doi: 10.1111/j.1365-4632.2006.02797.x

20. Tunc R, Saip S, Siva A, Yazici H. Cerebral venous thrombosis is associated with the major vessel disease Behçet's disease. Ann Rheum Dis. (2004) 63:16934. doi: 10.1136/ard.2003.018515

21. Alpsoy E, Uzun S, Akman A, Acar MA, Memisoglu HR, Basaran E. Histopathological and immunofluorescence findings of non-follicular lesions in patients with Behçet's disease. J Eur Acad Dermatol Venereol. (2003) 17:521-4. doi: 10.1046/j.1468-3083.2003.00625.x

22. Ilknur T, Pabuççuoglu K, Akin C, Lebe B, Gunes AT. Histopathologic and direct immunofluorescence findings of the papulopustular lesions in Behçet's disease. Eur J Dermatol. (2006) 16:146-50.

23. Ergun T, Gurbuz O, Dogusoy G, Mat C. Histopathological features of the spontaneous pustular lesions of Behçet's syndrome. Int J Dermatol. (1998) 37:194-6. doi: 10.1046/j.1365-4362.1998.00276.x

24. Jorizzo J, Abernethy JL, White WL, Mangelsdorf HC, Zouboulis CC, Sarica R, et al. Mucocutaneous criteria for diagnosis of Behçet's disease: an analysis of clinicopathologic data from multiple international centers. J Am Acad Dermatol. (1995) 32:968-76. doi: 10.1016/0190-9622(95)91 333-5

25. Jennette JC, Falk RJ, Bacon PA, Basu N, Cid NC, Ferrario F, et al. 2012 revised interntional chapel hill consensus conference nomenclature of vasculitides. Arthritis Rheumatol. (2013) 65:1-11. doi: 10.1002/art.37715

26. Sarica-Kucukoglu R, Akdag-Kose A, Kayabali M, Yazganoglu $\mathrm{KD}$, Disci R, Erzengin D, et al. Vascular involvement in 
Behçet's disease: a retrospective analysis of 2319 cases. Int J Dermatol. (2006) 45:919-21. doi: 10.1111/j.1365-4632.2006. 02832.x

Conflict of Interest: The authors declare that the research was conducted in the absence of any commercial or financial relationships that could be construed as a potential conflict of interest.
Copyright (C) 2021 Nakamura, Tsunemi, Kaneko and Alpsoy. This is an open-access article distributed under the terms of the Creative Commons Attribution License (CC $B Y)$. The use, distribution or reproduction in other forums is permitted, provided the original author(s) and the copyright owner(s) are credited and that the original publication in this journal is cited, in accordance with accepted academic practice. No use, distribution or reproduction is permitted which does not comply with these terms. 